\title{
Valenztheoretische Analyse des Hydrotops
}

\author{
Von ULRICH A. CORTI
}

(Mitteilung aus der Eidg. Anstalt für Wasserversorgung, Abwasserreinigung und Gewässerschutz an der Eidg. Technischen Hochschule in Zürich).

\section{A. EINLEITUNG}

Als Hydrotop (Wasserfeld, «Aquarium» s. 1.) bezeichnen wir einen endlichen, z. B. durch das Erdreich als Behälter (Schale, Gefäß) und durch die Atmosphäre als «offene Bedeckung» begrenzten, von Wasser erfüllten Raum, einschließlich alle weiteren darin befindlichen Elemente.

Sofern der Hydrotop von Bakterien, Pflanzen, Protozoen, Mollusken, Krustazeen, Insekten, Fischen usw. bewohnt wird, ist er als Biotop (Lebensraum) gekennzeichnet.

Der Hydrotop läßt sich kategorial und integral in drei Hauptvalenzfelder differenzieren, in welchen die im Wasser lebenden Organismen oder die an diesem Medium bzw. Milieu irgendwie «interessierten» übrigen Lebewesen sämtliche oder auch nur einen Teil ihrer Vitalfunktionen entfalten.

\section{Als Biotop umfaßt der Hydrotop:}

I. Elemente (Faktoren), welche bestimmten Lebewesen bezüglich der Entfaltung und der Erhaltung von Individuum und Art sowie für den Genuß des Daseins irgendwelche Dienste leisten bzw. dienlich sind. Sie stellen gesamthaft die Dienstmatrix (r. Hauptvalenzfeld) dar und seien je durch ein positives Vorzeichen gekennzeichnet.

II. Elemente (Faktoren), welche bestimmte Lebewesen in irgendeiner Hinsicht stören. Wir fassen sie im Begriffe der Störungsmatrix (2. Hauptvalenzfeld) zusammen und versehen sie je mit einem negativen Vorzeichen. 
III. Elemente (Faktoren), welche sich gegenüber bestimmten Lebewesen indifferent verhalten. Ihre Totalität nennen wir die Neutralmatrix (3. Hauptvalenzfeld); sie tragen je ein neutrales Vorzeichen.

Auf Grund der drei vorstehend erwähnten, valenzmäßig differenzierten, biotopologischen Elementgruppen läßt sich nun eine klare, systematischaxiologische, d. h. Wertanalyse der konkreten Hydrotope durchführen. Man erhält dabei als Ergebnis ein eigenartiges, in sich geschlossenes System der Hydrobiologie, wie es in dieser Form unseres Wissens bisher noch nicht vorgelegt worden ist.

Die nachstehende Übersicht kann hinsichtlich der angeführten Beispiele aus naheliegenden Gründen keinerlei Anspruch auf Vollständigkeit erheben. Sie beschränkt sich vielmehr darauf, ein Netz (Grundschema) für die Eingliederung der sehr mannigfaltigen Gegebenheiten zu skizzieren. Immerhin sind in diesem Netz, um die für evolutive Zwecke notwendige Koordination von Wissenschaft und Praxis schärfer zu beleuchten, zahlreiche Maschen bereits «ausgefüllt» worden.

Letzten Endes soll wohl die hydrologische und hydrobiologische Forschung ebenso wie die ihr mehr oder weniger parallel laufende Technik dazu dienen, die Hilfsmatrix des Lebendigen, und insbesondere des Menschen, auf ein optimales bzw. ideales Niveau zu heben, die Störungsmatrix dagegen zu neutralisieren.

\section{B. DIE DIENSTMATRIX DES HYDROTOPS}

\section{Dienstmatrix und Mensch}

Die Gewässer (Hydrotope) der Erde stellen im ganzen genommen so gut wie unerschöpfliche Vorratskammern für Nahrungsmittel des Menschen dar. Hier sei nur kurz auf die Bedeutung des Fleisches der wasserbewohnenden Wirbeltiere (z. B. Wale, Robben, Wasservögel, Schildkröten, Frösche, Fische), der Seewalzen (Holothurien), Seeigel (Echnoidea), Muscheln, Tintenschnecken (Zephalopoden), Schnecken, Krebse, Würmer (z. B. Palolowurm) sowie auf den Nähr- bzw. Genußwert mancher Wasserpflanzen (Tange, Wassernuß, Knollen von Sagittaria, Brunnenkresse usw.) hingewiesen. Dazu kommen noch das Trinkwasser und das Kochsalz.

Zahlreiche Nahrungsmittel, die aus den Gewässern bezogen werden, genießen, zum Teil allerdings erst nach passender Zubereitung, den Ruf 
von Delikatessen (Sardinen, Haifischflossen, Kaviar, Nuoc Mam - eine indochinesische Speisewürze aus Fischen -, Austern, Garnelen, Hummer, Langusten usw.). Mineralwasser dienen als Erfrischungsgetränke.

Mancherlei Organe und Produkte von Wassertieren hat der Mensch als Schmuck und Zierat verwenden gelernt. Dazu gehören unter anderem Pelze von Säugern (Seehundsfelle, Otterpelze,) Federn von Wasservögeln (Grebenfelle, Schwanenpelze, Schmuckfedern von Eiderenten), Krokodilleder, Schildpatt, Fischschuppen (Herstellung von sog. "Perlenessenz», Verwendung für kunstgewerbliche Zwecke), Perlen, Perlmutter, Korallen, Schnecken- und Muschelschalen als Zierat, Glasschwämme als Haarschmuck usw. Bekannt sind die Vorschläge zur Gewinnung von Gold aus Meerwasser.

Aus Haifischgalle, Purpurschnecken, Sepien werden oder wurden Farbstoffe gewonnen; die Ambra des Pottwals dient als geschätzter Riechstoff.

Im menschlichen Genußleben spielen sodann die Haltung von Zieraquarien, Robben als Zirkustiere, Wasserspiele, die allgemeine Ästhetik der Gewässer, der Wasser- und Angelsport, die Jagd auf Wassertiere, das Badeleben usw. eine keineswegs geringe Rolle.

Als Futtermittel dienen unter anderem Eingeweide von verschiedenen Wassertieren, ferner Wasservögel, Fische, Muscheltiere, verschrotete Garnelen, auch Daphnien, Tubifiziden u. a.m. Fische, Weichteile von Muscheln, Pierwürmer usw. werden als Angelköder benützt. Der Guano der Seevögel, der sog. Granatguano (Garnelen), Seesterne, Krabben- und Muschelschalen sowie die Asche von Seetangen liefern Düngemittel. Jod-, Brom- und andere Salze aus Meerwasser finden ebenso wie das Hirudin der Blutegel und der Lebertran Verwendung in der Medizin. Hinreichend bekannt ist die heilende Wirkung der Mineralquellen, der Thermal-, Mineral-, Meer- und Solbäder. Aus Os sepiae sind früher Zahnpulver bereitet worden.

Besonders vielseitig ist die Nutzung der Hydrotope für Zwecke der menschlichen Technik. So dienen die Gewässer der Errichtung von Wasserkraftanlagen (Gewinnung mechanischer, thermischer, elektrischer Energie usw.), einer Fülle von Verkehrszwecken, als Bezugsquellen für Wasch-, Spül-, Koch-, Lösungs- und andere Arten von Brauchwasser, zur Erzeugung von Dampf, Eis und zur Isolierung von chemischen Produkten. Speziell erwähnt werden möge etwa noch die Verwendung von Wasserpflanzen, wie Schilfrohr, als Streue- und Baumaterial, von Spongien als Badeschwämme, von Os sepiae als Poliermittel und Werkstoff(Meerschaum), 
von Kieselgur (Radiolarien, Diatomeen) als Träger von Katalysatoren und Nitroglyzerin (Dynamit) sowie als Filterhilfsmittel, von Perlmutter, Fischbein, Schildpatt, Elfenbein der Walroßzähne als Werkstoffe, von Rochenstacheln zur Anfertigung von Pfeilspitzen, von Muschelschalen zur Herstellung von Gefäßen, Messern, Schabern, Bohrern sowie von Geräten zur Tonerzeugung. Eiderdaunen dienen als Füllmaterial von Kissen, Schalen der Kaurischnecke als Zahlungsmittel, Fischöle als Brennstoffe für Heiz- und Beleuchtungszwecke, Austernschalen zur Herstellung von Luminophoren und Muschelschalen überhaupt als Mittel zur Beschotterung von Wegen. Hausenblase, Agar-Agar und Carragheen-Moos liefern Leim bzw. Geliermittel und Nährböden für Bakterienkulturen.

Schließlich leisten einige Tiere dem Menschen Dienste als Gesundheitspolizei (Aasvernichtung durch Wasservögel, Ausmerzung schwächlicher Fische durch Hechte), andere als Jagdgehilfen (Kormorane) oder als Wetteranzeiger. In der Kunst spielen Wasserpflanzen und -tiere sowie die Gewässer ganz allgemein als Vorlagen und Modelle, in der Mystik als Symbole eine Rolle. Endlich verdienen zahlreiche Elemente der Hydrotope Erwähnung als Sammel-, Handels- und Forschungsobjekte.

Die vorstehende, durchaus kursorische Aufstellung von Beispielen für die Wertrelationen zwischen Mensch und Hydrotop läßt bereits klar erkennen, wie umfassend der Bereich der von den Lebensfunktionen unseres Homo sapiens beschlagnahmten Umweltelemente ist. Sie zeigt weiter, daß sich alle diese positiven Elemente in nur zwei Grundkategorien einteilen lassen, nämlich in solche, welche

a) der Entfaltung und Erhaltung von Individuum und Art sowie

b) dem Verlangen nach und der Steigerung bzw. Vermehrung von lustbetontem Erleben der Welt dienen.

Es ist kaum zu erwarten, daß der weitere Ausbau des Systems derjenigen Hydrotopelemente, welche sich in bezug auf den Menschen durch eine positive Valenz auszeichnen, die Aufstellung einer dritten oder gar noch weiterer Grundkategorien erfordert.

Die Technik beruht ganz allgemein auf separatorischen und kombinatorischen Operationen; sie wandelt durch Analyse s. str. (Teilung, Auflösung) oder durch Synthese s. str. (Verbindung, Aufbau) die naturgegebenen Komponenten des Hydrotops in eine Form um, die eine unmittelbare Nutzung durch den Menschen ermöglicht, sofern die erwähnten Komponenten einer solchen Nutzung nicht schon an sich zugänglich sind. 
Eine wesentliche Aufgabe der Forschung (die erst zu Wissenschaft im eigentlichen Sinne führt) besteht darin, die Natur, in unserem besonderen Falle die Hydrotope, der Technik (als Naturbeherrschung) zu erschließen; auf eine zweite Aufgabe wird im Abschnitt «Störungsmatrix und Mensch» einzutreten sein. Nicht zu übersehen ist, $\mathrm{da} ß$ die Forschung im Dienste der Wissenschaft und Technik dem Forscher oft angenehme Sensationen vermittelt, ein Umstand, dem meist kaum Beachtung geschenkt wird.

\section{Dienstmatrix und Tier}

In bezug auf die Tierwelt lassen sich prinzipiell durchaus analoge, valenztheoretische Argumente anwenden, wie sie für den Menschen aufgezeigt wurden.

Die in den Gewässern lebenden Tiere ernähren sich teils ausschließlich von Pflanzen oder Tieren, teils von gemischter Kost. Viele Arten nehmen auch Schlamm, Detritus, Schwimm- oder Treibgut, Abfallstoffe des menschlichen Haushaltes (Abwässer!) usw. als Nahrungsmittel auf. Sodann ernähren sich auch manche amphibisch lebende und Trockengelände bewohnende Tiere, wenigstens zeitweise, von aquikolen Organismen.

$\mathrm{Ob}$ die Aufnahme gewisser in den Gewässern vorhandener Organismen bei den Verzehrern lustbetonte (= angenehme) Sensationen auslöst, läßt sich zwar vermuten, jedoch streng wissenschaftlich nicht beweisen.

Das Medium Wasser dient den im Hydrotop lebenden Tieren als Bewegungs-, Verkehrs-, Spiel-, Schutzraum, den Fortpflanzungselementen (Spermien, Eier usw.) als Transportmittel. Als solche kommen für die passive Dislokation bekanntlich auch Tiere, Treibgut, Schiffskörper usw. in Frage.

Der Hydrotop weist in der Regel eine Menge von Warten auf, die als Siesta-, Ernte-, Jagd-, Putz-, Sicherungs-, Kopulationswarten usw. dienen können. Dazu gehören z. B. der Gewässergrund, Steine, Mauerwerk, Holzpfähle, Metallpfeiler, die Wasseroberfläche und das Milieu interne des Wassers, Wasserpflanzen, Tiere, Treibgut. Entsprechende Warten leisten Dienste als Laichträger.

Ferner finden sich normalerweise in jedem Hydrotop Refugien (Felshöhlen, Schlupfwinkel unter Steinen und zwischen Korallenriffen, Kolke, Wasserpflanzendickichte, Schlammfelder, Sandgrund usw.) vor.

Im Wasser dienen die Licht-, Temperatur-, Druck-, Strömungs- und Konzentrationsverhältnisse (Chemismus) als indirekte Orientierungsmittel. Die Erzeugung von Differenzen in der Beschaffenheit des Mediums seitens der Wassertiere liefert Signalmittel. 
Im Bereiche der Fortpflanzungssphäre sind als Hilfsmittel der aquikolen Tiere beim Nestbau Steine, Sandkörner, Schlammpartikel, das Kalzium und andere Elemente des Wassers, Wasserpflanzen oder Teile von solchen, Treibgut usw. zu betrachten.

Grundsätzlich, d. h. soweit erkennbar, stehen sämtliche von den in Gewässern lebenden Tieren als Hilfsmittel (Dienstmatrix) verwendeten bzw. verwerteten Elemente des Hydrotops ausschließlich im Dienste der Entfaltung und Erhaltung von Individuum und Art, eine Feststellung von trivialem Charakter, die jedoch wegen ihrer «Durchsichtigkeit» erwähnt werden muß.

\section{Dienstmatrix und Wasserpflanze}

Für den Unterhalt der Wasserpflanzen fallen als Elemente der Dienstmatrix des Hydrotops unter anderem in Betracht: Die Tragkraft des Wassers und die jeder Pflanzenart adäquate Beschaffenheit des Untergrundes (für Wurzler und Hafter), der Gehalt des Milieus an Nährstoffen, das Vorhandensein von Licht, Wärme, Kohlendioxyd, Sauerstoff usw. Abgestorbene Organismen liefern Düngemittel. Viele Tiere (z. B. Wasservögel) tragen zur (endozoischen) Verbreitung von Hydro- bzw. Heleophyten bei; Wind, Wasserströmungen, Insekten führen die Bestäubung bzw. Befruchtung zahlreicher Wasserpflanzen herbei. (Auf die Dienstmatrix der Bakterien soll hier nicht näher eingetreten werden; sie eröffnet keine prinzipiell neuen Aspek te.)

Wie von den Tieren, werden also bei objektiver Betrachtungsweise auch von den Wasserpflanzen gewisse Elemente des Hydrotops in den Dienst der Entfaltung und Erhaltung von Individuum und Art gestellt.

\section{DIE STÖRUNGSMATRIX DES HYDROTOPS}

\section{Störungsmatrix und Mensch}

Neben den positiven Elementen der Dienstmatrix enthält der Hydrotop eine Fülle von Elementen (Faktoren), welche den Menschen in mehr oder weniger gravierender Weise stören bzw. gefährden können. Auch hier kann es nicht unsere Aufgabe sein, alle möglichen Störungs- bzw. Gefährdungsmomente aufzuzählen. Wir greifen nur wenige Beispiele heraus.

Das Wasser vermag durch UUberschwemmungen (intensive Niederschläge, Dammbrüche, Sturmfluten) Schäden an Bauwerken, Kulturen und Menschen anzurichten. Der Verkehr auf, in und mit dem Wasser ist prinzi- 
piell mit Gefahren verbunden (Unfälle). Auf die korrodierende Wirkung des Wassers sei nur beiläufig hingewiesen; Bohrmuscheln haben schon größere Bauwerke im Wasser zerstört.

Der Genuß von verdorbenen Fischen, Krebsen, Muscheln usw., auch solchen, die von Parasiten befallen sind, kann zu Erkrankungen oder Vergiftungen führen. Gewisse Arten von Fischblut wirken toxisch. Das Eindringen spitziger Stacheln oder Dornen von Wassertieren in Haut- und Muskelgewebe oder in die Blutbahn führt mitunter zu heftigen Entzündungen. Auch Bisse von Krokodilen, Haien usw. schließen Gefahren ein. Mitunter entstehen erhebliche Schäden dadurch, daß Wassertiere Fischfangeinrichtungen zerstören. Verwesende Wasserpflanzen und -tiere geben oft zu argen Geruchsbelästigungen Anlaß, ebenso Guanolager, ungereinigte Abwässer, Schwefelquellen usw. Verunreinigte, ungepflegte Gewässer bilden Gefahrenherde für Gesundheit und Wohlfahrt (Malaria!), auch stören sie das ästhetische Empfinden.

Faßt man die Gesamtheit der den Menschen störenden Faktoren (Elemente) der Hydrotope ins Auge, so ergibt sich auch hier eine Verteilung auf zwei Grundkategorien. Die negativ zu wertenden Hydrotopelemente lassen sich gliedern in solche, welche:

a) die Entfaltung und Erhaltung von Individuum und Art verhindern, partiell hemmen oder wenigstens zeitweise stören;

b) zu unlustbetonten (unangenehmen) Welterlebnissen führen.

Aufgabe der Forschung und T'echnik ist es, diejenigen Maßnahmen zu treffen, welche die Störungsmatrix ausschalten oder ihre Wirkung auf ein Minimum reduzieren, derart, daß auch die unlustbetonten Welterlebnisse verschwinden.

Die im Abschnitt «Dienstmatrix und Mensch» angedeutete zweite Aufgabe der Forschung besteht darin, Mittel und Wege zu suchen, welche die unangenehme Seite der inneren (psychischen), durch Neugierde, Sensationshunger, Nichtwissen, Leiden bedingte Unruhe im Menschen zu tilgen ermöglichen. Es ist zu bekannt, daß der Forscher sehr oft Enttäuschungen erfährt, welche die Ursache von unlustbetonten Sensationen bilden.

\section{Störungsmatrix und Tier}

Die Zahl derjenigen Faktoren des Hydrotops, welche in irgendeiner Weise störend in das Leben der aquikolen Tiere eingreift, ist außerordentlich groß. Es gehören dazu nicht nur die aktiv störenden Effektoren des aquatischen Milieus, sondern auch die Mangelverhältnisse des letzteren, 
z. B. Sauerstoffdefizit, Nahrungsmangel, Fehlen von Geschlechtspartnern, von Refugien, Licht, ausreichendem Lebensraum usw.

Ferner spielen Temperatur-, Druck-, Lichtextreme, extremer Wellengang und Chemismus des Wassers, Gifte, starke Versandung und Verschlammung des Grundes, Geschiebeprozesse, das Auftreten intensiver Trübungen, die Verunreinigung des Mediums durch Abwasser, stark variierende Strömung, Vereisung, Verlandungs- und Austrocknungsvorgänge, heftige Niederschläge (z. B. Hagel), Gewitter, die Bildung von Staub-, Schaum-, Öl- und Fettschichten an der Wasseroberfläche, Hindernisse (z. B. Wasserpflanzendickichte, Wehre), starke Eutrophierung, pflanzliche und tierische Parasiten, Verfolger usw. eine mehr oder minder wichtige Rolle als Störungsfaktoren.

\section{Störungsmatrix und Wasserpflanze}

Auch die Hydro- bzw. Heleophyten sind vor zahlreichen Störungsfaktoren des Hydrotops nicht völlig geschützt.

Intensive Wasserbewegung kann zur Entwurzelung von Pflanzen oder zur Loslösung von Haftern führen; viele Pflanzen werden von Tieren verzehrt, von Parasiten befallen, durch Geschiebe zermalmt, durch Schleim eingehüllt. Versandungs- und Verschlammungsprozesse sowie Austrocknungsvorgänge können zum Absterben der Wasserpflanzen führen, desgleichen die Veränderung des Milieuchemismus durch Abwasser oder infolge des Eindringens von Meerwasser in Süßwasser. Andere Störungsmomente bestehen in der Vereisung der Gewässer, in Mangel an Licht, Kohlendioxyd, Sauerstoff, Nährsalzen, im Auftreten von Hochwasser (Heleophyten!). Unter den vom Festlande (Trockengelände) her drohenden Störungsfaktoren seien nur die Eingriffe des Menschen erwähnt (Rodung, Abmähen der Wasserpflanzen, Trockenlegung von Gewässern).

\section{ALLGEMEINE ERGEBNISSE}

\section{DER VALENZTHEORETISCHEN ANALYSE}

\section{DES HYDROTOPS}

Unsere, vielleicht etwas langatmige, Aufzählung von Beispielen verfolgte zunächst den Zweck, die eminent bunte Mannigfaltigkeit der Sachverhalte, welche der Hydrotop umfaßt, aufzuzeigen und dieselben in ein einfaches System zu bringen. Dieses System erhellt, daß eine rein wert- 
mäßige, d. h. valenztheoretische oder axiologische Analyse des Hydrotops grundsätzlich sämtliche wissenschaftlichen wie technischen Probleme der Hydrologie und Hydrobiologie aufzurollen erlaubt, sofern auch das - in der vorliegenden Abhandlung nicht zur Darstellung gelangte - Milieu interne der Organismen in die Untersuchungen einbezogen wird. Auch im Milieu interne lassen sich die «biologischen Wertigkeiten» der einzelnen Organe, Organsysteme und Organelemente valenztheoretisch analysieren. Als singuläres Beispiel sei nur der Wert des Verdauungstraktus für die Entfaltung und Erhaltung des Individuums angeführt. Besonders interessant sind namentlich die valenzmäßigen Korrelationen zwischen Elementen des Milieu interne und Milieu externe (z. B. Verdauungstraktus und exogene Nahrung!). Eine vollständige Analyse sämtlicher Wertrelationen ergibt als Resultante eine umfassende Hierarchie der biotypischen Werte. Dieselbe beleuchtet zudem die primordiale Bedeutung des Problems der «Lebenswerte». Der Biotop verwandelt sich damit in einen Axiotop (Wertfeld).

Es zeigt sich, daß für jedes Lebewesen, abgesehen von den lebensnotwendigen Elementen des Milieu interne, eine gewisse, hinsichtlich Zahl, Dimension, Struktur, Ordnung usw. von Art zu Art und meist auch von Individuum zu Individuum verschiedene Gruppe von Elementen des Milieu externe eine essentielle und damit komplementäre Rolle spielt. Daraus folgt, daß jedes lebendige Individuum ein «offenes System» bildet; auf keinen Fall läßt es sich durch sein Integument gegenüber der sog. Umwelt definieren.

Eine integrale Analyse der Hydrotope setzt die Ermittlung sämtlicher vorhandenen und möglichen Wertrelationen voraus. Diese Aufgabe läßt sich mit gleichem Nutzen für die Wissenschaft und für die Technik lösen. Zieht man schließlich aus unseren Darlegungen die Quintessenz, so ergibt sich als die wohl zentralste aller biologischen Fragen diejenige nach dem existentiellen, subjektiven Eigenwert des einzelnen, lebendigen Individuums. Ihre Beantwortung würde den Rahmen unserer valenztheoretischen Hydrotopanalyse weit überschreiten. 